\section{Structural Asymmetry of AcrB Trimer Suggests a Peristaltic Pump Mechanism}

\author{
Markus A. Seeger, ${ }^{1,3 *}$ André Schiefner, ${ }^{2 *} \dagger$ Thomas Eicher, ${ }^{1}$ François Verrey, ${ }^{1}$ \\ Kay Diederichs, ${ }^{2}$ Klaas M. Pos ${ }^{1} \ddagger$
}

The AcrA/AcrB/TolC complex spans the inner and outer membranes of Escherichia coli and serves as its major drug-resistance pump. Driven by the proton motive force, it mediates the efflux of bile salts, detergents, organic solvents, and many structurally unrelated antibiotics. Here, we report a crystallographic structure of trimeric AcrB determined at 2.9 and 3.0 angstrom resolution in space groups that allow asymmetry of the monomers. This structure reveals three different monomer conformations representing consecutive states in a transport cycle. The structural data imply an alternating access mechanism and a novel peristaltic mode of drug transport by this type of transporter.

$\mathrm{D}$ rug resistance during infection or cancer treatment is often caused by the overproduction of efflux transporters, leading to decreased levels of antibiotics or chemotherapeutics inside the cells $(1,2)$. Drugefflux transporters can be of the $\mathrm{ABC}$ type that use the free energy of adenosine triphosphate (ATP) hydrolysis, or they can be secondary transporters that use the proton motive force to energize the extrusion of drugs. ABC-type transporters are predominantly found in eukaryotes, whereas in prokaryotes the main drug-efflux systems function as $\mathrm{H}^{+} /$drug exchangers. In Gram-positive bacteria, drug resistance is often conferred by members of the major facilitator superfamily (3). In Gram-negative bacteria, however, resistancenodulation-cell division (RND) type efflux pumps play a dominant role $(1,4)$. Structures of the primary membrane transport proteins bacteriorhodopsin (5-7) and the $\mathrm{Ca}^{2+}$-ATPase
(8) in different conformations allowed to establish hypotheses about a solute transport pathway. Transport of solutes by secondary carriers of the major facilitator superfamily [LacY (9) and GlpT (10)] and the glutamate transporter homolog $\mathrm{Glt}_{\mathrm{Ph}}(11-13)$ is likewise expected to depend on major structural conversions (14).

The first structure of the RND pump AcrB was obtained at $3.5 \AA$ resolution from crystals grown in a trigonal space group assigned as R32 (15-18). An AcrB monomer contains 12 transmembrane $\alpha$ helices (TM1 to TM12) (fig. $\mathrm{S} 1)$. TM4 and TM10 are surrounded by the other transmembrane helices of the monomer and harbor the residues D407, D408 (TM4), and K940 (TM10), which appear to play an essential role in proton translocation $(16,19)$. The periplasmic part of AcrB consists of the TolC

Fig. 1. Main structural differences of the AcrB monomers. (A) The three AcrB monomers shown in top view as cylinder presentation in blue (L), yellow $(\mathrm{T})$, and red (0) are superimposed onto the symmetric AcrB trimer model depicted in transparent gray. In the T monomer (yellow), a hydrophobic pocket is defined by phenylalanines 136, 178, 610, 615, 617, and 628; valines 139 and 612; isoleucines 277 and 626; and tyrosine 327 at the PN2/PC1 interface. (B) Structural changes in the putative proton translocation site. Conserved residues D407, D408 (TM4), and K940 (TM10) in the three monomers (L, blue; $T$, yellow; 0 , red) are depicted with 2Fo-Fc electron density maps contoured at $0.5 \sigma$ (L) or $1 \sigma$ (T and 0 ) as viewed from the cytoplasm. In the $\mathrm{L}$ and $\mathrm{T}$

A

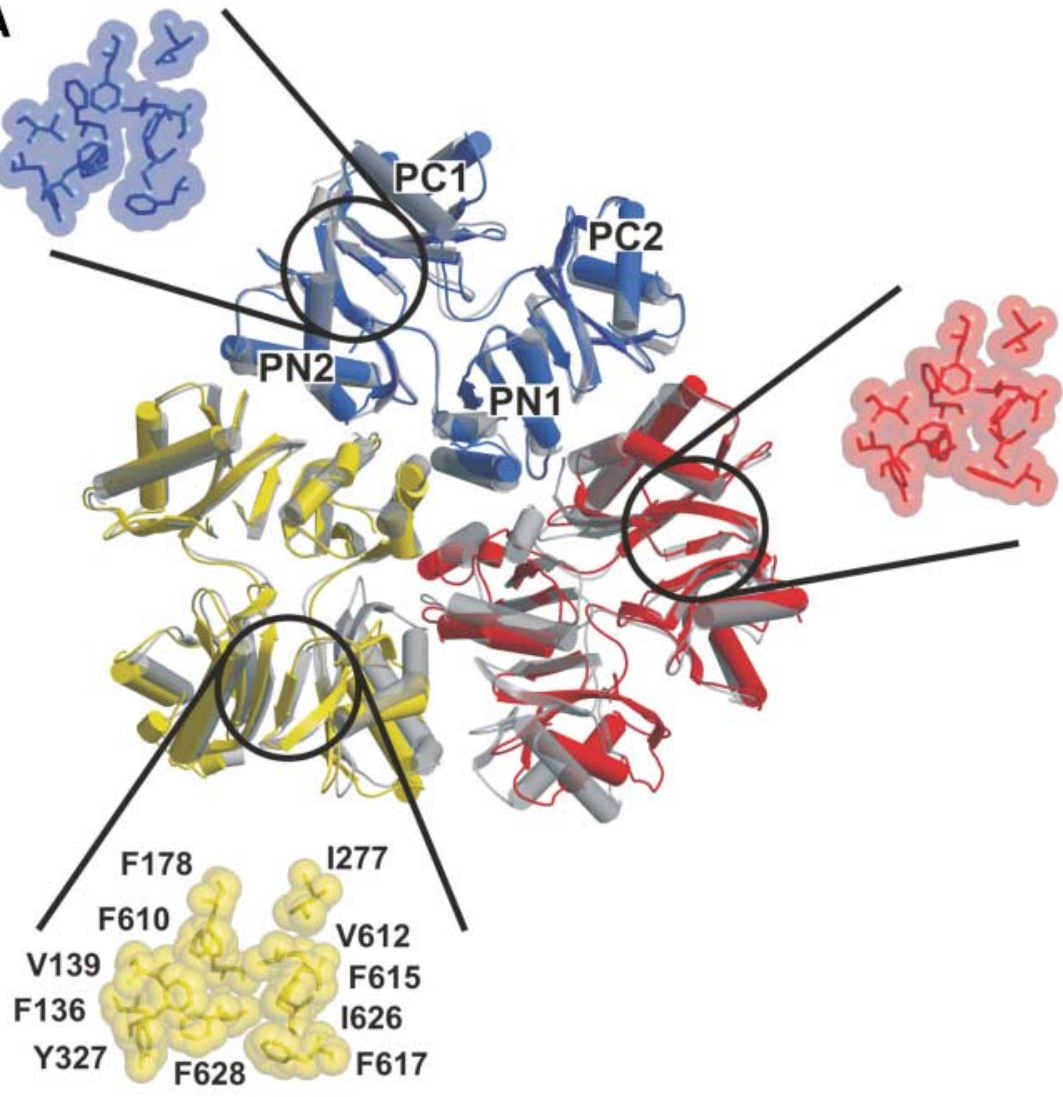

${ }^{1}$ Institute of Physiology and Zurich Centre for Integrative Human Physiology (ZIHP), University of Zurich, Winterthurerstrasse 190, Zürich, Switzerland. ${ }^{2}$ Department of Biology, University of Konstanz, Universitätsstrasse 10, M647, D-78457 Konstanz, Germany. ${ }^{3}$ Institute of Microbiology, Swiss Federal Institute of Technology (ETH), Zürich, Switzerland.

*These authors contributed equally to this work.

†Present address: Department of Molecular Biology, BCC206, The Scripps Research Institute (TSRI), 10550 North Torrey Pines Road, La Jolla, CA 92037, USA.

$\ddagger$ To whom correspondence should be addressed. E-mail: kmpos@access.unizh.ch monomers, the same conformation is observed, whereas in the 0 monomer, K940 forms a salt bridge with D407. This interaction seems to be stabilized by hydrogen bonding of T978 (TM11). To restore the geometry as it appears in the L monomer, proton uptake is anticipated.

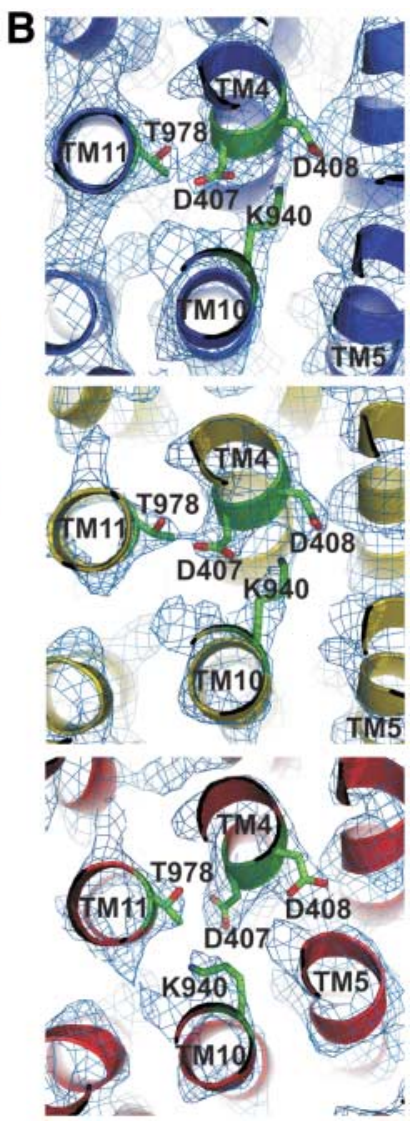


docking domain (DN and DC subdomains), which is located farthest from the membrane plane, and the pore domain, composed of subdomains PN1, PN2, PC1, and PC2. The TolC docking domain, on which TolC has been shown to dock coaxially (20), exhibits a funnel-like structure narrowing to a central pore located in the pore domain. The central pore structure consists of three $\alpha$ helices (designated pore helices) donated by the PN1 subdomains of each AcrB monomer. Near the membrane plane, the central pore leads to a central cavity, and further to a $35 \AA$ wide transmembrane hole defined by the ringlike arrangement of the TM helices of the trimer, which was proposed to be filled with phospholipids (16). Three vestibules at the monomer interfaces located just above the membrane plane lead toward the central cavity. The current hypothesis about the mechanism of transport envisions the diffusion of substrates via the transmembrane domains and vestibules into the central cavity and the opening of the central pore to allow the transport of the substrates through AcrB toward TolC (21) and export to the external medium $(16,22)$. Large changes were postulated to be associated with this transport function, which was tentatively termed "elevator mechanism" (17), but remained elusive because of a lack of structural information.
Here, we define the structural changes that constitute the basis of solute transport by AcrB. To gain structural insight into the mechanism of transport by AcrB, crystals belonging to monoclinic $(C 2,2.9 \AA$ resolution) and triclinic $(P 1$, $3.0 \AA$ A resolution) space groups were grown, and complete data sets with good statistics were measured (table S1). Previous functional interpretations $(16-18,23)$ were based on the trigonal crystal form, which harbors a trimer with exact three-fold symmetry. In this crystal form, models with good geometry and low crystallographic $R$ factor have not been reported so far. Our subsequent analysis revealed that those crystals that appeared to be $R 32$ suffered from merohedral twinning and were in fact twinned $R 3$ crystals with noncrystallographic symmetry (table S1). A high twinning fraction results in a blurred electron density, which cannot be computationally deconvoluted, and explains the difficulties encountered with the trigonal crystal form. We therefore analyzed the data from the untwinned $C 2$ and $P 1$ crystal forms containing three and six monomers corresponding to one and two nonsymmetric trimers, respectively.

The structures of these three independently obtained asymmetric trimers are very similar (table S2); distances given below refer to the asymmetric trimer from the well-diffracting $C 2$ crystal form. Its atomic model (Fig. 1) was refined to $\mathrm{R}$ factors of $R=22.6 \%\left(R_{\text {free }}, 26.7 \%\right)$ (table S1). It is substantially more complete than the model based on the trigonal crystal form and contains one consecutive chain of amino acids 2 to 1033 in two monomers and of amino acids 2 to 1045 in the third monomer. The C-terminal amino acids 1034 to 1057 (1046 to 1057) are unassigned as a result of missing electron density.

At root mean square deviations (RMSD) between 2.1 and $3.1 \AA$ (table S2), the monomers of a trimer structurally deviate from each other much more than can be expected from monomers obeying noncrystallographic symmetry (Fig. 1 and fig. S2). One monomer appears not to be constrained by interaction with its neighbors, and its conformation is therefore termed loose (L). Another exhibits an opening from the inside of the pore domain toward the funnel of the AcrB trimer, and its conformation is designated as open $(\mathrm{O})$. In this monomer, the PN1 subdomain is tilted toward and tightly interacts with the neighboring monomer's PN1 and PN2 subdomains. This interaction imposes a constraint on the conformation of the neighboring monomer, which we label tight $(\mathrm{T})$. The conformation of the L monomer is closest (RMSD of $0.9 \AA$ ) (table S2) to that of the symmetric AcrB monomer model, whereas the $\mathrm{T}$ and $\mathrm{O}$ monomers
Fig. 2. Visualization of tunnels in the pore domain of the AcrB peristaltic drug efflux pump. Each monomer shows a tunnel penetrating the periplasmic part in a distinct manner. The tunnels are highlighted as green surfaces in a ribbon model of the AcrB trimer. The upper panels shows the side view and the lower panels the top view of the AcrB trimer. (A) In the L monomer (blue), the tunnel starts at the lateral cleft (PC1/PC2 interface) $\sim 15 \AA$ above the membrane plane extending halfway toward the center (pore) of the trimer. (B) In the T monomer (yellow), the tunnel extends diagonally upward through the pore domain (PN1/PN2 interface) toward the pore in the center of the trimer. (C) With the conversion from $T$ to 0 , the PC1/PC2 cleft closes because of the conformational change of the PC2 subdomain and leads to complete closure of the tunnel laterally. Moreover, tilting of the PN1 subdomain in the $\mathbf{O}$ monomer creates an exit pathway toward the funnel and TolC.
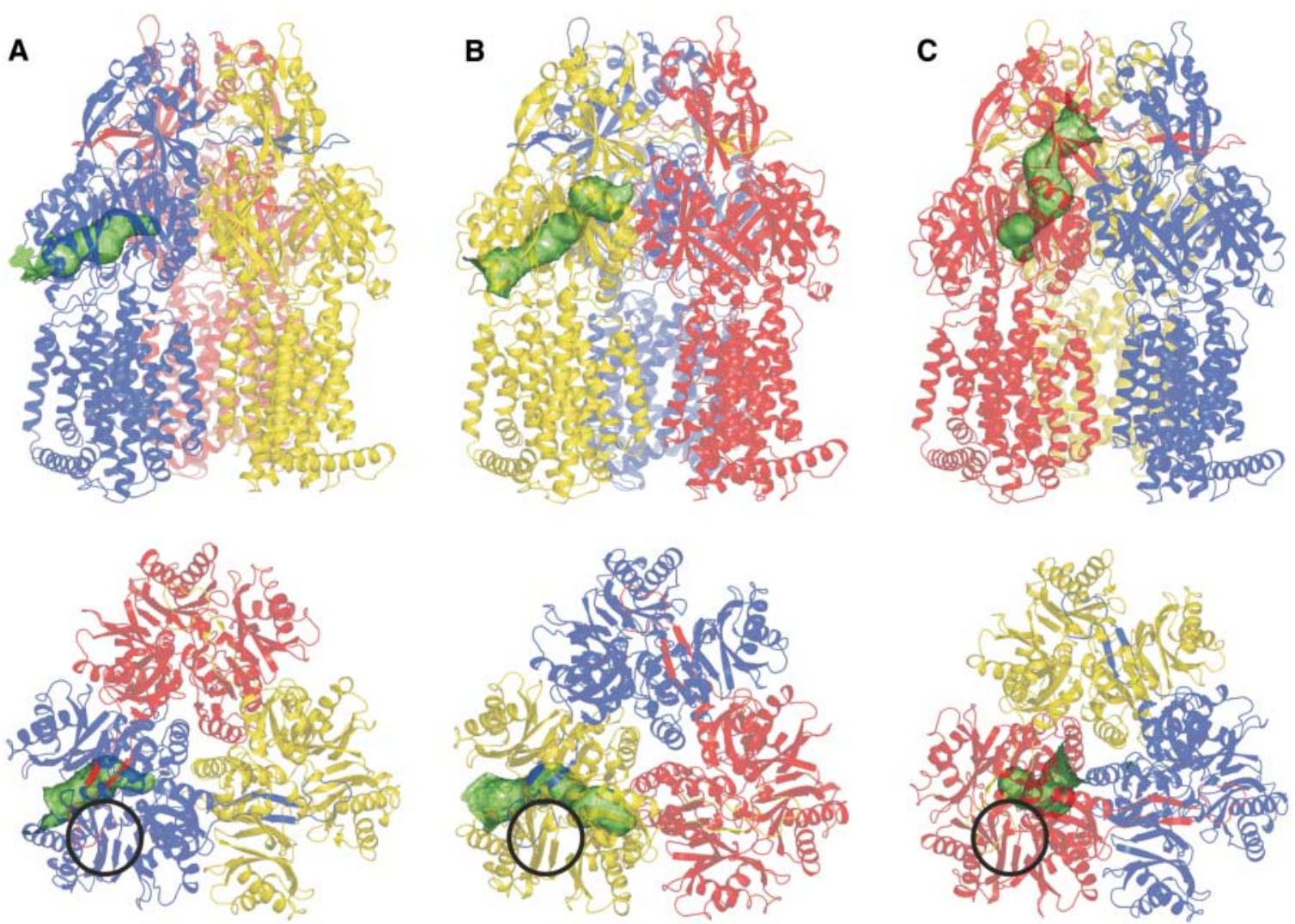

The location of the hydrophobic binding pocket in the T monomer (yellow) is indicated in each conformation by a black circle. Only in the T monomer is this binding pocket accessible for substrates. 
differ significantly from it (RMSD of 1.8 and $3.0 \AA$, respectively) (table S2).

The largest structural changes are located in the pore domain of the trimer, whereas in the TolC docking domain, including the intermonomer connecting loops, no major structural differences are detected (fig. S2). A prominent feature of the AcrB trimer is the periplasmic central "pore," composed of PN1 subdomain $\alpha$ helices (Fig. 1A). Compared with the orientation of the other PN1 subdomains, the PN1 subdomain of the $\mathrm{O}$ monomer is considerably tilted $\left(\sim 12^{\circ}\right)$ toward the PN1 and PN2 subdomains of the T monomer (Fig. 1A). A synchronous reorientation of the $\mathrm{PC} 2$ subdomain mediated through an antiparallel $\beta$-sheet (N $\beta 2$ $\mathrm{C} \beta 13$ ) interaction (fig. S1) in the $\mathrm{O}$ monomer reduces the distance between the PC2 subdomain and the membrane plane by more than $6.5 \AA$ (figs. S2 and S3). A coil-to-helix transition and bending of the helical $\mathrm{N}$-terminal part of TM8 appears to be associated with this large conformational change. This part of TM8 adopts a coil conformation in the $\mathrm{L}$ monomer and forms an intermediate conformation in the $\mathrm{T}$ monomer and a kinked helix in the $\mathrm{O}$ monomer (fig. S3B). In the $\mathrm{T}$ monomer, the $\mathrm{PC} 2$ subdomain does not change in comparison to the L-monomer conformation, most probably because the PN1 subdomain (and therefore the PC2 subdomain) is locked because of interaction with the $\mathrm{O}$ monomer's PN1 subdomain (Fig. 1A). A contraction of the $\mathrm{T}$ monomer is observed as a result of the movement of its TM helices (except TM2) of up to $3 \AA$ toward its periplasmic part (fig. S2) and appears to be induced by the upshift of the PN1 subdomain of the T monomer because of interaction with the PN1 subdomain of the $\mathrm{O}$ monomer (Fig. 1). From the PN1 subdomain, the upshift is transduced to TM3 and TM4 by two parallel $\beta$ sheets, $N \beta 1^{\prime}$ and Nß16 (fig. S1).

The structural changes in the $\mathrm{T}$ monomer create a hydrophobic pocket in the PN2/PC1 subdomain interface (Fig. 1A), which is not present in the $\mathrm{L}$ and $\mathrm{O}$ monomers. We suggest that the hydrophobic pocket located on the $\mathrm{T}$ monomer may supply a substrate binding pocket inside the pore domain (Fig. 1A).

Visualization of cavities within the protein revealed large tunnels in the pore domain of all three monomers (Fig. 2). The width of these tunnels would be sufficient to accommodate solute molecules transported by AcrB. In the $\mathrm{L}$ and $\mathrm{T}$ monomers, a large, laterally accessible pathway is present about $15 \AA$ above the membrane plane. In vivo, AcrA (24) is expected to protect the lateral exit against direct contact with the periplasm $(16,25,26)$. In the $\mathrm{T}$ monomer, the tunnel leads to residues of the $\mathrm{O}$ monomer PN1 subdomain, which operates as a plug for the tunnel exit. In the O conformation, the tilting of the PN1 subdomain opens an exit pathway from the binding pocket toward the funnel of AcrB. Because of the simultaneous structural change of the PN1 and PC2 subdomains, the lateral entrance of the tunnel observed in the $\mathrm{L}$ and $\mathrm{T}$ monomer is completely closed in the $\mathrm{O}$ monomer and determines the direction of substrate transport toward the funnel (Fig. 2C).

The asymmetric structure of AcrB suggests that the three monomer conformations represent consecutive states of a transport cycle. A possible drug-transport mechanism can be proposed if a cycling of each monomer through the conformations $\mathrm{L}, \mathrm{T}, \mathrm{O}$, and back to $\mathrm{L}$ is assumed. The PN1 subdomains, including the pore helices, might play the role of a ratchet pin that enforces the order of the conformational changes. The PN1 conformational change from $\mathrm{O}$ to the $\mathrm{L}$ monomer causes the loss of strong interaction with the PN1 and PN2 subdomains of the $\mathrm{T}$ monomer and enables the conversion of the PN1/PC2 subdomains from $\mathrm{T}$ to the $\mathrm{O}$ monomer conformation. Consequently, bound substrate in the $\mathrm{T}$ monomer would be squeezed out of the hydrophobic pocket located at the interface of the $\mathrm{PN} 2 / \mathrm{PC} 1$ subdomains during the transition to the $\mathrm{O}$ monomer conformation. The conversion of the PN1 subdomain of the T monomer into the tilted conformation of the $\mathrm{O}$ monomer also reestablishes strong interaction with the PN2 subdomain of the L monomer and induces conformational changes leading to formation of the binding pocket at the PN2/PC1 interface (Fig. 1) and the change from the $\mathrm{L}$ to the $\mathrm{T}$ monomer conformation.

This sequence of events creates a pathway for the efflux of drugs through the tunnels with a transport mechanism that is analogous to that of a peristaltic pump and is schematically displayed in Fig. 3. Diffusion of substrate within the tunnel is limited by an occlusion site whose position migrates toward the funnel, effectively guiding the substrate toward TolC. The unspecific nature of transport implied by such a mechanism could account for the observed broad substrate specificity of the AcrA/AcrB/TolC
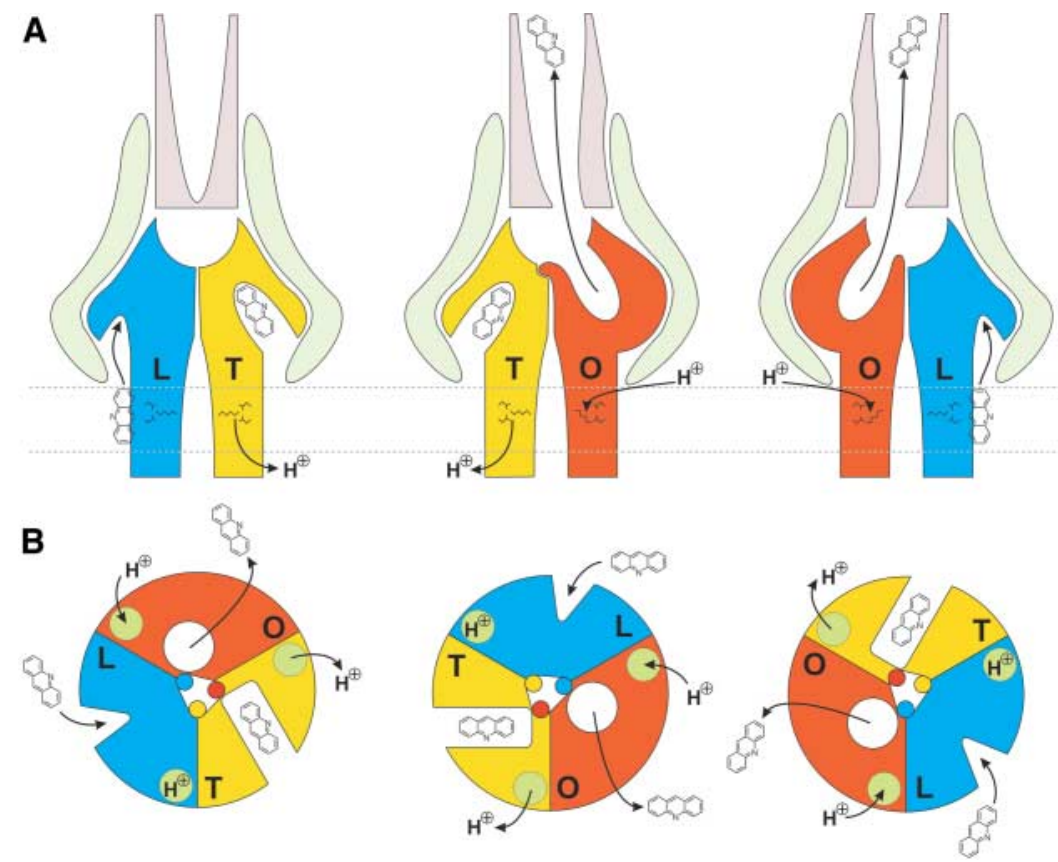

Fig. 3. Schematic representation of the AcrB alternating site functional rotation transport mechanism. The conformational states loose (L), tight $(\mathrm{T})$, and open $(\mathrm{O})$ are colored blue, yellow and red, respectively. (A) Side-view schematic representation of two of the three monomers of the AcrB trimer. AcrA and TolC are indicated in light green and light purple colors, respectively. The proposed proton translocation site (D407, D408, and K940) is indicated in the membrane part of each monomer. (B) The lateral grooves in the L and T monomer indicate the substrate binding sites. The different geometric forms reflect low (triangle), high (rectangle), or no (circle) binding affinity for the transported substrates. The PN1 subdomains (including the pore helices) located in the middle of the model are highlighted and form the corners of an asymmetric triangle (white) to indicate the communication between the monomers. In the first state of the cycle, a monomer binds a substrate (acridine) in its transmembrane domain (L conformation), subsequently transports the substrate from the transmembrane domain to the hydrophobic binding pocket (conversion to $\mathrm{T}$ conformation) and finally releases the substrate in the funnel toward TolC ( 0 conformation). The conversion from the 0 -monomer to the L-monomer conformation is suggested to be the major energy-requiring (proton motive force-dependent) step in this functional rotation cycle and requires the binding of a proton to the proton translocation site (D407, D408, and K940) from the periplasm. The conversion from the $\mathrm{T}$ monomer to the $\mathrm{O}$ monomer is accompanied by the release of a proton from the proton translocation site to the cytoplasm. AcrA can be expected to participate in the transduction of the conformational changes from AcrB to TolC, which results in the opening of the TolC channel and the facilitation of drug extrusion to the outside of the cell. 
pump as well as for the transport of small molecules such as hexane $(27,28)$. It is therefore proposed that besides the specific binding in the hydrophobic pocket of the T monomer, unspecific diffusion of small substrates through the observed tunnels occurs. Substrate transport from the membrane domain to the periplasmic tunnel might be via a lateral pathway, including the TM8/TM9 groove and the AcrA/AcrB interface. Recent reports $(25,26)$ substantiate the close interaction between AcrA and AcrB (or of the homolog proteins MexA and MexB) in the tripartite complex, and we therefore anticipate conformational changes of AcrA coupled to those of AcrB. On the other hand, our structure does not support substrate transport through the central pore, as implied by the elevator mechanism $(14,17)$, because access of substrates from the central cavity to the funnel is prohibited by the small diameter of the pore.

As AcrB is energized by the proton-motive force, transient protonation of titratable groups within the transmembrane domain of the protein can be expected to be the mechanism that delivers the energy required for the conformational changes described above. Indeed, we observe a prominent K940 (TM10) side-chain reorientation away from D408 and toward D407 (both on TM4) in the O conformation (Fig. 1B) and a bulging of TM5 toward TM4 and TM10, strengthening the hypothesis that this part of the transmembrane domain is central to proton binding and release $(16,19)$. As thoroughly investigated in the case of bacteriorhodopsin $(5-7,29)$, side-chain rearrangements (Fig. 1B) can leverage global conformational changes of the magnitude we observed for TM8 (fig. S3), involving reversible tilts of helices and the transient change of kinks and bulges in the main chain. We speculate that, in the case of AcrB, subtle changes in the transmembrane part (TM4 and TM10) produce the large conformational changes in the pore domain ultimately resulting in drug efflux. Different from what has been suggested for LacY (30) and EmrE (31), the proton and substrate translocation in AcrB appear to be spatially separated.

We have proposed a possible transport mechanism that, based on a functional rotation of the trimer, creates a peristaltic pump mechanism in each monomer (Fig. 3). Our model merges Jardetzky's alternate access pump (32) with the rotating site catalysis of $\mathrm{F}_{1} \mathrm{~F}_{0}$-ATPase $(33,34)$ and suggests a working hypothesis for the transport mechanism of RND transporters.

\section{References and Notes}

1. X. Z. Li, H. Nikaido, Drugs 64, 159 (2004).

2. S. V. Ambudkar, I. W. Kim, Z. E. Sauna, Eur. J. Pharm. Sci. 27, 392 (2006)

3. P. N. Markham, A. A. Neyfakh, Curr. Opin. Microbiol. 4 509 (2001).

4. K. Poole, Clin. Microbiol. Infect. 10, 12 (2004).

5. J. K. Lanyi, Mol. Membr. Biol. 21, 143 (2004).

6. H. Luecke, Biochim. Biophys. Acta 1460, 133 (2000).

7. R. Neutze et al., Biochim. Biophys. Acta 1565, 144 (2002).

8. C. Toyoshima, G. Inesi, Annu. Rev. Biochem. 73, 269 (2004).

9. ]. Abramson et al., Science 301, 610 (2003).

10. Y. Huang, M. J. Lemieux, ]. Song, M. Auer, D. N. Wang, Science 301, 616 (2003).

11. D. Yernool, O. Boudker, Y. Jin, E. Gouaux, Nature 431, 811 (2004).

12. D. J. Slotboom, I. Sobczak, W. N. Konings, J. S. Lolkema, Proc. Natl. Acad. Sci. U.S.A. 96, 14282 (1999)

13. M. Grunewald, B. I. Kanner, J. Biol. Chem. 275, 9684 (2000).

14. I. Sobczak, ]. S. Lolkema, Curr. Opin. Microbiol. 8, 161 (2005).

15. K. M. Pos, K. Diederichs, Acta Crystallogr. Biol. Crystallogr. D58, 1865 (2002).

16. S. Murakami, R. Nakashima, E. Yamashita, A. Yamaguchi, Nature 419, 587 (2002).

17. E. W. Yu, G. McDermott, H. I. Zgurskaya, H. Nikaido, D. E. Koshland Jr., Science 300, 976 (2003).
18. K. M. Pos, A. Schiefner, M. A. Seeger, K. Diederichs, FEBS Lett. 564, 333 (2004)

19. L. Guan, T. Nakae, J. Bacteriol. 183, 1734 (2001).

20. N. Tamura, S. Murakami, Y. Oyama, M. Ishiguro, A. Yamaguchi, Biochemistry 44, 11115 (2005).

21. V. Koronakis, A. Sharff, E. Koronakis, B. Luisi, C. Hughes, Nature 405, 914 (2000).

22. S. Murakami, N. Tamura, A. Saito, T. Hirata, A. Yamaguchi, J. Biol. Chem. 279, 3743 (2004)

23. E. W. Yu, ]. R. Aires, G. McDermott, H. Nikaido, J. Bacteriol. 187, 6804 (2005)

24. J. Mikolosko, K. Bobyk, H. I. Zgurskaya, P. Ghosh, Structure 14, 577 (2006).

25. D. Nehme, K. Poole, Antimicrob. Agents Chemother. 49, 4375 (2005).

26. T. Touze et al., Mol. Microbiol. 53, 697 (2004).

27. D. G. White, J. D. Goldman, B. Demple, S. B. Levy, J. Bacteriol. 179, 6122 (1997).

28. X. Z. Li, L. Zhang, K. Poole, J. Bacteriol. 180, 2987 (1998)

29. B. W. Edmonds, H. Luecke, Front. Biosci. 9, 1556 (2004).

30. O. Mirza, L. Guan, G. Verner, S. Iwata, H. R. Kaback, EMBO J. 25, 1177 (2006).

31. N. Gutman, S. Steiner-Mordoch, S. Schuldiner, J. Biol. Chem. 278, 16082 (2003).

32. 0. Jardetzky, Nature 211, 969 (1966).

33. J. P. Abrahams, A. G. Leslie, R. Lutter, ]. E. Walker, Nature 370, 621 (1994).

34. P. D. Boyer, Annu. Rev. Biochem. 66, 717 (1997).

35. We thank the beamline staff at the Swiss Light Source (SLS) of the Paul Scherrer Institut in Villigen (Switzerland), and at the European Synchrotron Radiation Facility (ESRF) in Grenoble (France). This work was supported by a grant of the Transregio-Sonderforschungsbereich ZurichKonstanz to K.D. We thank P. A. Karplus and W. Welte for critical reading and discussion. The atomic coordinates and structure factors of the $\mathrm{C} 2$ and P1 crystal forms have been deposited at the Protein Data Bank with accession numbers 2 GIF and $2 \mathrm{HRT}$, respectively. This work is dedicated to Peter Dimroth on the occasion of his 65th birthday.

\section{Supporting Online Material}

www.sciencemag.org/cgi/content/full/313/5791/1295/DC1

Materials and Methods

Figs. S1 to S3

Tables S1 and S2

References

20 June 2006; accepted 1 August 2006

$10.1126 /$ science. 1131542 